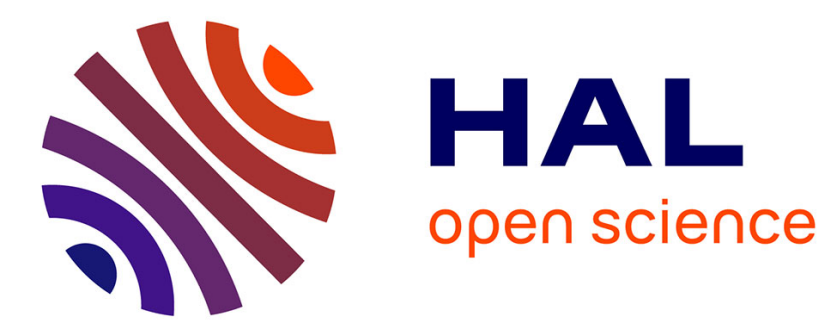

\title{
Détecteur de rapport pour un récepteur monocanal application à un monochromateur à simple faisceau
}

Amouyeli, Blet, Riollet

\section{To cite this version:}

Amouyeli, Blet, Riollet. Détecteur de rapport pour un récepteur monocanal application à un monochromateur à simple faisceau. Revue de Physique Appliquée, 1966, 1 (1), pp.74-76. 10.1051/rphysap:019660010107400 . jpa-00242688

\section{HAL Id: jpa-00242688 https://hal.science/jpa-00242688}

Submitted on 1 Jan 1966

HAL is a multi-disciplinary open access archive for the deposit and dissemination of scientific research documents, whether they are published or not. The documents may come from teaching and research institutions in France or abroad, or from public or private research centers.
L'archive ouverte pluridisciplinaire HAL, est destinée au dépôt et à la diffusion de documents scientifiques de niveau recherche, publiés ou non, émanant des établissements d'enseignement et de recherche français ou étrangers, des laboratoires publics ou privés. 


\title{
DÉTECTEUR DE RAPPORT POUR UN RÉCEPTEUR MONOCANAL APPLICATION A UN MONOCHROMATEUR A SIMPLE FAISCEAU
}

\author{
Par MMOUYEL, BLET et RIOLLET,
}

Laboratoire de Physique du Solide, Compagnie Française Thomson-Houston.

\begin{abstract}
Résumé. - Lorsque l'on veut mesurer les variations d'une grandeur par rapport à une référence en fonction d'un paramètre, on utilise de préférence un appareil à deux canaux dont on enregistre le rapport des indications. Le dispositif que nous présentons ici permet d'utiliser un appareil monocanal grâce à une exploration alternée et une mémoire électromécanique asservie à la grandeur de référence. L'application particulière décrite correspond à la transformation d'un monochromateur optique simple en spectrophotomètre automatique.
\end{abstract}

Abstract. - In order to measure the variation of some quantity as a function of one parameter with respect to a reference, one ordinary make use of a double channel instrument recording the ratio of the two values. We present here a new system, which allow us to make use of a single channel apparatus owing to an alternating exploration and an electromechanical memory triggered to the reference value. The special application described below corresponds to the modification of a single beam optical monochromator in a automatic spectrophotometer.

Principe. - Nous supposons que nous voulons déterminer les variations, en fonction d'un paramètre, du rapport d'une grandeur " échantillon » $E(x)$ à une grandeur de référence $R(x)$ cette dernière étant fonction du paramètre $x$ soit :

$$
y=E(x) / R(x) \text {. }
$$

L'appareil utilisé étant par définition monocanal ne peut nous donner que l'une ou l'autre des valeurs $E(x)$ ou $R(x)$. Il est facile de concevoir, selon la nature de la grandeur à étudier, un dispositif électrique, mécanique ou optique permettant de faire des mesures alternées à une cadence déterminée. Cette cadence doit répondre à certaines obligations : être assez lente devant la constante de temps de réponse du récepteur et être assez rapide par rapport aux variations de $E(x)$ et de $R(x)$.

Si nous enregistrons alors le signal de sortie du récepteur, le graphique se présentera sous la forme de deux courbes tiretées reliées entre elles par les traces d'aller et retour de style ( $f g .1$ ).

L'enregistreur ayant en général une vitesse uniforme, les deux courbes représentent les variations de $E$ et de $R$ en fonction du temps par l'intermédiaire de :

$$
x=F(t) .
$$

La grandeur finale que l'on cherche étant le rapport :

$$
E(x) / R(x)
$$

il convient pour chaque abscisse (en $t$ ) de mesurer chacune des deux ordonnées, d'en faire le rapport et de tracer la nouvelle courbe.
C'est ce travail manuel, cause d'erreur, long et fastidieux, que nous avons voulu remplacer par un asservissement mémorisé permettant l'enregistrement direct du rapport.

L'opération se fait en deux temps: les deux phases de l'exploration alternée.

Première phase : Exploration de la référence. Le récepteur délivre un signal proportionnel à $R(x)$. Soit $V_{1}=k R(x)$. Soient $V_{\mathrm{M}}$ et $V_{\mathrm{m}}$ les valeurs maximale et minimale de ce signal dans le domaine exploré. Prenons une tension de référence $V_{0}$ au plus égale à $V_{\mathrm{m}}$. Appliquons le signal de sortie $V$, en tête d'un potentiomètre. Un servomoteur relié à une chaîne d'asservissement classique ajuste la tension du curseur à $V_{\mathbf{0}}$.

Le signal à enregistrer, prélevé sur ce curseur est donc égal au signal " référence " $V_{1}$ multiplié par le facteur :

$$
V_{0} / V_{1}
$$

Deuxième phase : Exploration de l'échantillon. La tension de sortie du récepteur est devenue $V_{2}=k E(x)$. Bloquons l'asservissement pendant cette phase, le multiplicateur du signal de sortie reste : $V_{0} / V_{1}$ et le signal de sortie prélevé toujours sur le curseur du potentiomètre est égal à :

$$
V_{2} \times V_{0} / V_{1} \text {. }
$$

On enregistre donc une grandeur proportionnelle à :

$$
V_{2} / V_{1} \text { soit } E(x) / R(x) \text {. }
$$

En outre l'existence d'un diviseur entre curseur du potentiomètre et masse permet de comparer 


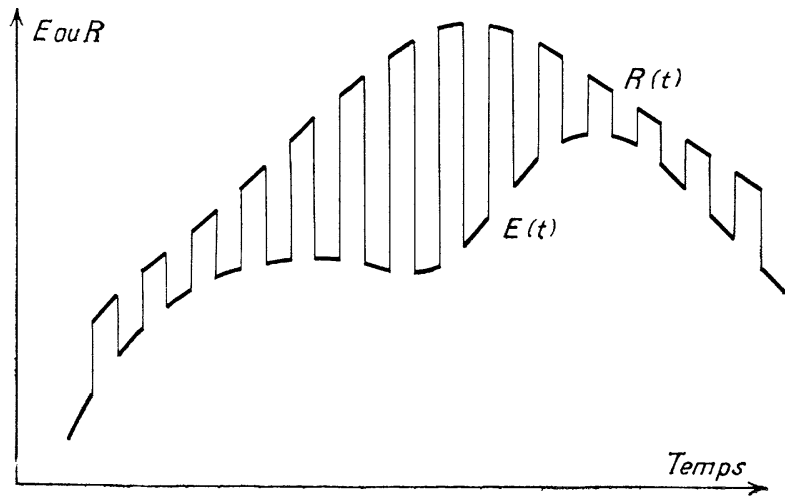

FIg. 1. - Enregistrement alterné avec présentation intermittente de l'échantillon.

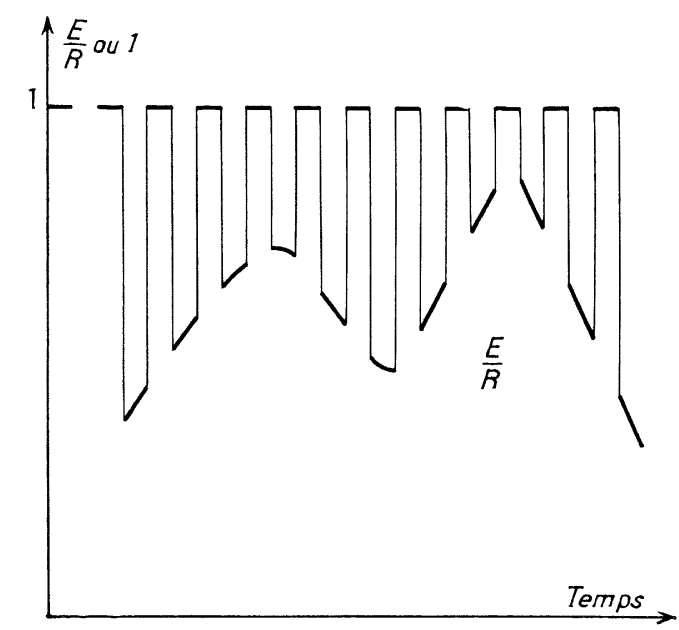

Fig. 2. - Même enregistrement que sur la figure 1 mais " normalisé " par le circuit d'asservissement. directement : $V_{2} / V_{1}$ à $V_{0} / n$, ce qui est très intéressant lorsque $V_{2} / V_{1}$ est petit.

L'enregistrement se traduit donc comme précédemment par deux courbes tiretées, l'une ayant une ordonnée constante égale à $V_{0}$, ou $V_{0} / n$ avec diviseur, l'autre ayant l'ordonnée variable

$$
V_{0} \times V_{2} / V_{1}=V_{0} \times E(x) / R(x) .
$$

Cette seconde courbe, comparée à la première donnera donc par lecture directe la valeur de $E(x) / R(x)(f i g .2)$.

Exemple d'application. - Nous allons indiquer l'application que nous avons faite de ce principe pour mesurer directement sans calculs la courbe du facteur de transmission d'un filtre coloré au moyen d'un monochromateur (à un seul faisceau).

A) Partie optique. - Le monochromateur est un modèle Terrien-Desvignes double additif soustractif à prismes de fluorine et miroirs. La source à spectre continu est une lampe à incandescence à filamient de tungstène, le récepteur est un photomultiplicateur.

B) Partie mécanique. - Étant donné la petite dimension des filtres colorés à l'étude $(4 \times 6 \mathrm{~mm})$ nous avons dû les examiner en lumière convergente (l'ouverture du monochromateur est de F/6) en plaçant le filtre juste devant la fente d'entrée. Un petit support à glissière entraîné par un électroaimant commandé par un moteur synchrone équipé d'une came permet l'introduction et l'escamotage alternés du filtre devant la fente avec une cadence convenable.

C) Partie électronique. - Le récepteur est nous l'avons dit un photomultiplicateur alimenté

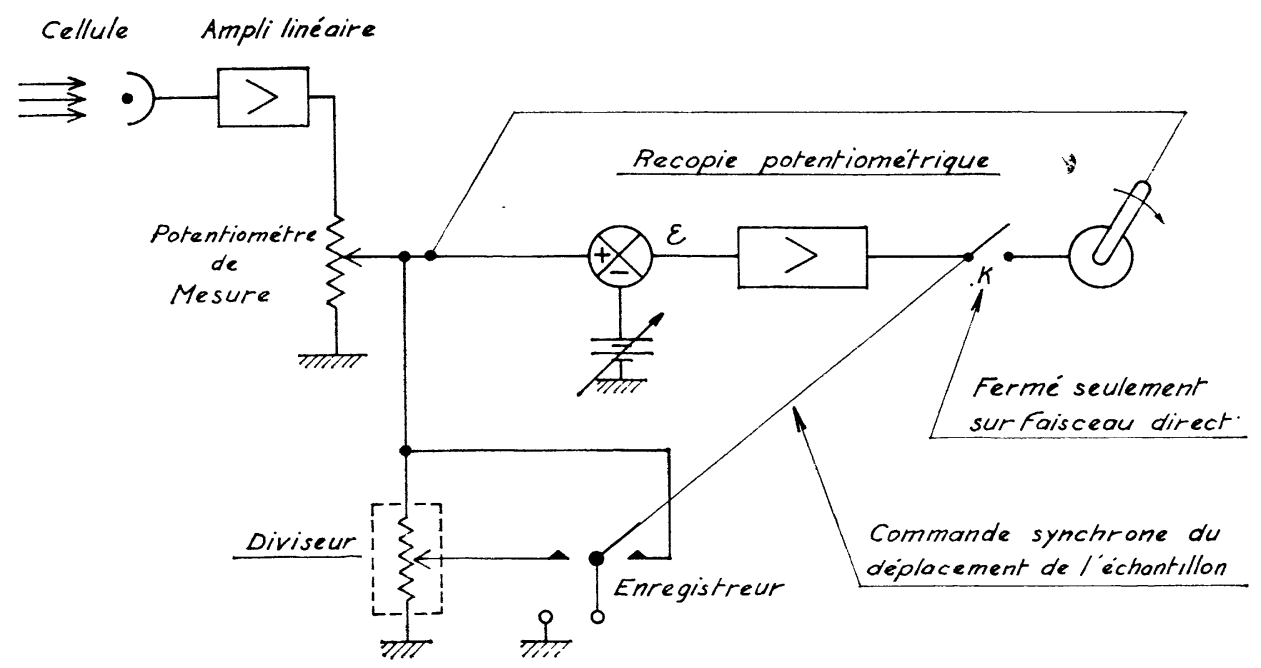

FIG. 3. - Schéma fonctionnel de l'asservissement mémorisé. 
par une tension stabilisée. Un amplificateur de courant convertisseur d'impédance est connecté dans le circuit d'anode. Le signal de sortie est envoyé dans un potentiomètre d'asservissement. Un amplificateur différentiel classique à courant continu compare la tension au curseur de ce potentiomètre avec une tension de référence. Un moteur d'asservissement branché à la sortie de cet amplificateur agit sur le curseur du potentiomètre de manière à rendre égales les tensions précédentes. Le schéma fonctionnel est représenté sur la figure 3. Mentionnons que le moteur qui assure la présentation intermittente de l'échantillon connecte en synchronisme la borne de sortie de l'ensemble alternativement sur le curseur du potentiomètre et sur une position convenable d'un diviseur. Il est ainsi possible de déterminer un facteur de transmission de faible valeur avec une grande précision, par comparaison. avec une valeur convenable $1 / n$.

L'utilisation pour potentiomètre d'asservissement d'un potentiomètre à variation exponentielle assure une précision et un amortissement constants. Bien

Fig. 4, 5 et 6 . - Courbes de transmission de filtres colorés utilisés en télévision en couleur.

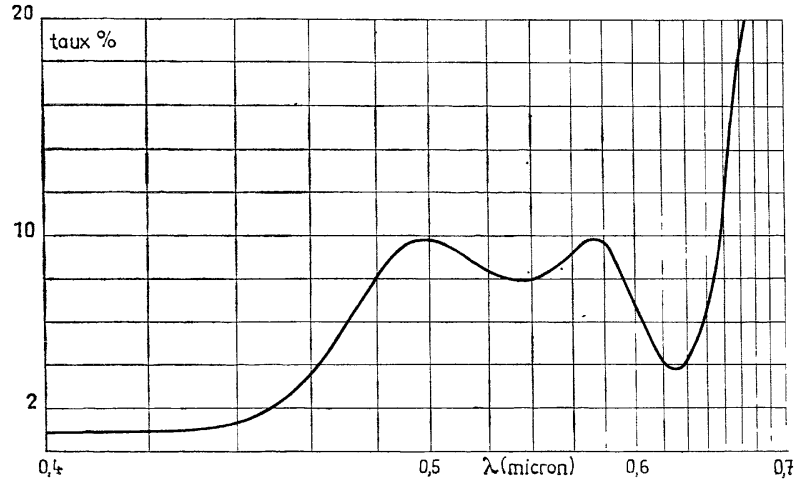

Fig. 4. - Filtre V. entendu pendant la phase de mesure l'amplificateur d'asservissement est court-circuité comme nous l'avons dit précédemment.

D) Résultats. - Ainsi qu'il résulte des quelques enregistrements reproduits ci-contre ( $f$ g. 4 à 6) on se rend compte que ce système a le double avantage

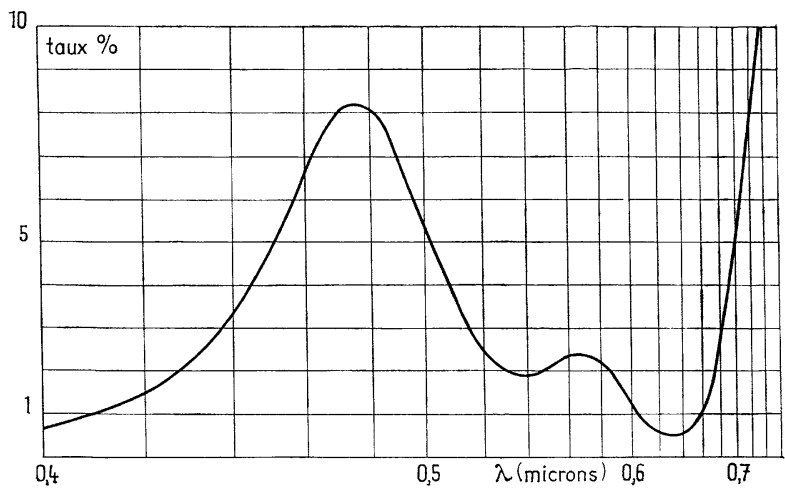

FIG. 5. - Filtre BV.

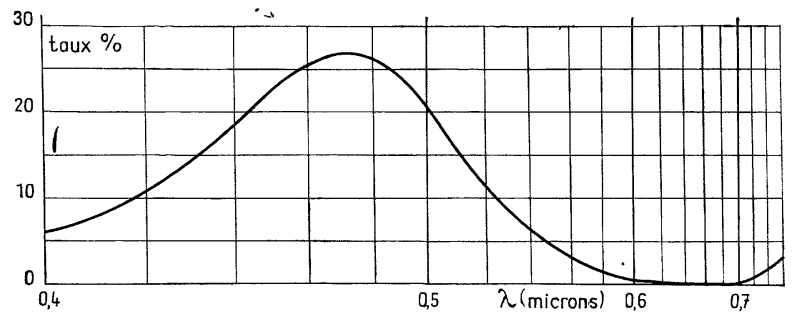

FIG. 6. - Filtre C.

de l'automaticité et de la précision. Par contre il faut reconnaître que son domaine d'utilisation est limité à l'enregistrement des phénomènes à variations lentes et continues, la comparaison n'étant pas simultanée, mais alternée.

Manuscrit reçu le 12 novembre 1965. 